\title{
Relationship Between Functional Residual Capacity and Oxygen Desaturation During Short Central Apneic Events During Sleep in "Late Preterm" Infants
}

\author{
PIERRE TOURNEUX, ANDRÉ LÉKÉ, GUY KONGOLO, VIRGINIE CARDOT, LOIC DÉGRUGILLIERS, KAREN CHARDON, \\ LAURENT STORME, GÉRARD KRIM, JEAN-PIERRE LIBERT, AND VÉRONIQUE BACH
}

Péritox (EA 4285-INERIS) [P.T., A.L., V.C., L.D., K.C., G.K., J.-P.L., V.B.], University of Picardy, Amiens 80036, France; Médecine
Néonatale et Réanimation Pédiatrique Polyvalente [P.T., A.L., G.K., L.D., G.K.], Service de Cardio-Pneumo-Allergologie Pédiatrique
[L.D.], Centre Hospitalier Universitaire, Amiens 80054, France; UPRES-JE2490 [L.S.], Université Lille II, Lille 59000, France

ABSTRACT: Apneic episodes are frequent in the preterm neonate and particularly in active sleep (AS), when functional residual capacity (FRC) can be decreased. Furthermore, FRC may be inversely correlated with the speed of blood- $\mathrm{O}_{2}$-desaturation. We evaluated the potential involvement of FRC in the mechanisms responsible for blood- $\mathrm{O}_{2}$-desaturation during short central apneic events $(>3 \mathrm{~s})$ in "late-preterm" infants and analyzed the specific influence of sleep state. Apneic events were scored in 29 neonates (postmenstrual age: $36.1 \pm 1.2 \mathrm{wk}$ ) during $\mathrm{AS}$ and quiet sleep (QS). FRC was measured during well-established periods of regular breathing. Apneas with blood- $\mathrm{O}_{2}$-desaturation (drop in $\mathrm{SpO}_{2}>5 \%$ from the baseline, lowest $\mathrm{SpO}_{2}$ during apnea: $91.4 \pm 1.8 \%$ ) were more frequent in $\mathrm{AS}$ than in QS, whereas no difference was seen for apneas without desaturation. The magnitude of the FRC did not depend on the sleep state. In AS only, there was a negative relationship between FRC and the proportion of apneas with desaturation. Even in late preterm infants who do not experience long-lasting apnea, blood- $\mathrm{O}_{2}$-desaturation during short apneic events is related (in AS but not QS) to a low baseline FRC. Sleep stage differences argue for a major role of AS-related mechanisms in the occurrence of these apneas. (Pediatr Res 64: 171-176, 2008)

$\mathrm{T}$ he functional residual capacity (FRC) forms part of the body's $\mathrm{O}_{2}$ reserves and is commonly equated to the lung volume at end-expiration. In the adult, the FRC is determined by the elastic recoil forces of the lungs and the chest wall. In the neonate, the end-expiratory lung volume is actively determined by the breathing pattern (1-4). During apnea, very frequent at this age, the ability to maintain an oxygen supply from the existing reserves will be determined by the size of the FRC, the existence of any underlying disorders affecting lung growth and ongoing changes in lung volume and mechanics.

A negative correlation is observed between FRC and arterial oxygen tension and blood- $\mathrm{O}_{2}$-desaturation speed (independently of the occurrence of apneic events) $(3,5)$. Moreover, because the preterm neonate breathes with a FRC close to the closing volume, the closure of a few regions of the lung could occur and induce intrapulmonary shunting (with normal pul-

Received June 25, 2007; accepted March 28, 2008.

Correspondence: Pierre Tourneux, M.D., Ph.D., PériTox (EA 3901-INERIS), University of Picardy, 3 rue des Louvels, F-80036 Amiens, France; e-mail: tourneux. pierre@chu-amiens.fr, pierre.tourneux@u-picardie.fr

This work was supported by the Picardy Regional Council and the French Ministry of Research. monary blood flow in unventilated areas) leading to rapid transcutaneous desaturation (6).

Apneas or irregular breathing in the neonate are associated with reduced FRC $(7,8)$. Similarly, a lower FRC is observed if measured within 2 min of an apneic pause (3). However, the link between apneic events with hypoxemia and FRC remains subject to debate, since Marshall and Kattwinkel (9) did not find any relationship between the incidence of idiopathic apneic events and FRC.

The higher frequency of apneic events commonly observed in active sleep (AS) may be related to a low FRC $(2,10)$, although a decrease in FRC is not always found in this sleep state $(7,11)$. These conflicting results may be explained not only by various FRC measurement techniques but also because breathing regularity (in addition to the potentially differential effects of sleep state) may have affected the data. However, FRC values did not significantly vary between regular versus irregular breathing patterns, which predominate during active and quiet sleep (QS) respectively (11). Unfortunately, apneic events with and without blood- $\mathrm{O}_{2}-$ desaturation were not distinguished.

Many studies have focused on pathologic apneic events (usually considered as those lasting $>20 \mathrm{~s}$ or associated with blood- $\mathrm{O}_{2}$-desaturation and/or bradycardia). However, there is a need to study the basic physiologic mechanisms underlying short apneic events, which can also be associated with $\mathrm{O}_{2}$ desaturation. In addition, late-preterm infants are generally not considered, even though their breathing control is less mature than in full-term infants (12).

Our hypothesis is that a low baseline FRC is related to the frequency of apneic events, especially those accompanied by blood- $\mathrm{O}_{2}$-desaturation, in sleeping "late-preterm" infants. Therefore, we evaluated the potential involvement of FRC in the mechanisms responsible for the occurrence of blood- $\mathrm{O}_{2}-$ desaturation during short central apneic events $(>3 \mathrm{~s})$ as a function of the sleep state. To take into account any specific sleep state influences, FRC determination was only performed

\footnotetext{
Abbreviations: AS, active sleep; FRC, functional residual capacity; QS, quiet sleep; $\mathbf{S p O}_{2}$, blood $\mathrm{O}_{2}$-saturation
} 
during well established, regular breathing in polygraphically scored active or QS.

\section{MATERIALS AND METHODS}

The study was performed on a preliminary trial including 24 neonates at postmenstrual age equivalent to those of late preterm infants. To confirm the relevance of the results, the sample size has been increased up to 29 neonates (10 boys and 19 girls, gestational age: $30.4 \pm 1.7 \mathrm{wk}$, birth weight: $1.341 \pm$ $0.331 \mathrm{~kg}$, postnatal age: $5.7 \pm 2.3 \mathrm{wk}$, body weight: $2.068 \pm 0.289 \mathrm{~kg}$ ). Our sample size was set at a convenient level with respect to the recruitment capacity. Before the study, three neonates did not receive prenatal steroids, 13 required mechanical ventilation, 12 and 10 had received oxygen or caffeine treatment, respectively. At the time of the study, all were healthy and breathing room air spontaneously. The protocol was approved by the Regional Ethics Committee and written informed consent was obtained from the parents.

The measurements were performed during a morning nap ( $>3 \mathrm{~h}$ long). The diaper-clothed neonate lays in the supine position in a closed incubator (Médipréma ISIS, Chambray-les-Tours, France). Since temperature changes can induce sleep and breathing disturbances (13), the study was performed at thermoneutrality $(13,14)$. The room temperature was kept constant at $25^{\circ} \mathrm{C}$. The incubator temperature was measured with a thermocouple (placed $10 \mathrm{~cm}$ above the center of the mattress), the rectal temperature was monitored with a thermistor probe (inserted $1 \mathrm{~cm}$ beyond the anal sphincter) and the mean skin temperature was averaged from abdominal and cheek skin temperatures (thermocouples; accuracy $\pm 0.10^{\circ} \mathrm{C}$; Bioblock, Illkirch, France). Body and air temperatures $\left({ }^{\circ} \mathrm{C}\right)$ were sampled at 10 -s intervals.

Sleep. Sleep states (AS, QS, and intermediate sleep) were scored (15) on the basis of two electroencephalograms and eye movements (accelerometer attached to an eyelid). Limb movements (accelerometers attached to the wrists and ankles) and the ECG were also recorded (polysomnograph Alice 4, Respironics, Nantes, France). The breathing rate was assessed by transthoracic impedance using the ECG patch electrodes. Oxyhaemoglobin saturation was continuously assessed from the transcutaneous-pulsed $\mathrm{O}_{2}$-saturation $\left(\mathrm{SpO}_{2}\right)$ (Oximax MAX-N, Tyco Healthcare Group LP, Nellcor Puritan Bennett Division, CA) placed on the right hand.

Apnea. Central apneic events were defined as respiratory pauses lasting more than $3 \mathrm{~s}(16,17)$. Blood- $\mathrm{O}_{2}$-desaturation was defined as a drop in $\mathrm{SpO}_{2}$ of more than $5 \%$ when comparing the mean value preceding the apneic event and the lowest value measured during the apneic event (18). Apneic episodes were described in terms of their frequency $\left(\mathrm{h}^{-1}\right)$, mean and maximum duration (s). The proportion of apneic events with desaturation was calculated as the ratio between their frequency and the overall frequency of apneic episodes (i.e., regardless of their blood- $\mathrm{O}_{2}$-desaturation status).

FRC. Functional residual capacity $\left(\mathrm{mL} \mathrm{kg}^{-1}\right)$ was measured during wellestablished episodes of AS and QS using a validated oxygen wash-in washout technique $(19,20)$. This method (which consists in rapidly increasing the proportion of inspired $\mathrm{O}_{2}$ from $21 \%-100 \%$ and maintaining it for a 30 -s period) is easy to use and sufficiently accurate for clinical applications, since FRC can be assessed with a bias below 5\% (19).

The circuit has already been described (21). It comprises a facemask (dead space $1 \mathrm{~mL}$ ) attached to a pneumotachograph (Statice Santé, Besançon, France, dead space $1.35 \mathrm{~mL}$ ). This system does not disturb the neonate's ventilatory or sleep patterns (21). A two-way computer controlled valve (dead space $1.18 \mathrm{~mL}$ ) could be opened either to the bag containing $100 \% \mathrm{O}_{2}$ (inspiration during the hyperoxic period) or to the incubator air (inspiration during the baseline period and expiration). Breath-by-breath inspired and expired volumes and end-cycles $\mathrm{O}_{2}$ concentration were measured continuously using a mass spectrometer (MGA-1100, GE Marquette Medical Systems, Milwaukee, WI).

FRC was calculated taking into account the $\mathrm{O}_{2}$ consumption (evaluated with the mean inspired and expired $\mathrm{O}_{2}$ fractions over the minute preceding the hyperoxic period), the mean blood $\mathrm{Hb}$ concentration, the mean change in $\mathrm{SpO}_{2}$ (from $96 \%$ at baseline to $100 \%$ during the test) and the total dead space of the apparatus (19).

FRC was assessed during regular breathing to analyze the specific sleep state effect and to rule out a possible effect of an irregular breathing pattern on FRC $(3,7)$. A period was scored as regular if the respiratory rate was free of sighing or apneic events and if synchronous chest and abdomen movements were present for the $3 \mathrm{~min}$ before and during the FRC measurement. The values were corrected by conversion into STPD units. FRC was averaged over two to three measurements in each sleep state (1) (maximum cumulative exposure oxygen: $3 \mathrm{~min}$ ), as long as the coefficient of variation was below $15 \%$ (to ensure good reproducibility). If only one test could be performed in a given sleep state, the FRC value was considered as missing value.
Respiratory parameters (respiratory rate, tidal volume, minute volume, inspiratory and expiratory times, and oxygen consumption) and heart rate were averaged over the 30-s baseline period preceding the hyperoxic period.

Statistical analyses. $t$ tests and analyses of variance were performed (Statview ${ }^{\circledR}$, Cary, NC). When F-values were significant, PLSD post hoc tests were used. Parametric regressions were always confirmed by Kendall's nonparametric correlation coefficient to check that the significance of the regressions was not accounted for by just a few points.

Unpaired $t$ tests compared the influences of low versus high baseline $\mathrm{SpO}_{2}$ levels on apneic events with desaturation.

The significance level was $p<0.05$. Indicative results were given when relevant. Data were expressed as means \pm SD.

\section{RESULTS}

Sleep states and incubator and body temperatures. The neonates were recorded for a mean duration of $113.9 \pm 25.4$ min. AS and QS corresponded to $67.6 \pm 7.7 \%$ and $23.9 \pm$ $8.0 \%$ of the total sleep time, respectively (with intermediate sleep being discarded).

There was no sleep state effect on incubator temperature (AS: $32.5 \pm 0.8 v s$ QS: $32.6 \pm 0.9^{\circ} \mathrm{C}, t_{26}=1.94$, NS), skin (AS: $36.4 \pm 0.3 v s$ QS: $36.4 \pm 0.3^{\circ} \mathrm{C}, t_{26}=0.42$, NS) and rectal temperature (AS: $37.0 \pm 0.3$ vs QS: $36.9 \pm 0.3^{\circ} \mathrm{C}$, $\left.t_{18}=0.13, \mathrm{NS}\right)$.

Cardiorespiratory events. About 1900 apneic events were analyzed. All lasted less than 20 s. All infants displayed apneic events without blood- $\mathrm{O}_{2}$-desaturation in AS, and 24 of the 29 in QS. Apneic events with desaturation were noted in 28 of 29 infants in AS and 12 of 29 in QS $\left(\chi_{1}^{2}=18.13\right.$, $p<0.001$ ) (Table 1).

The mean $\mathrm{SpO}_{2}$ for the apneic events without blood- $\mathrm{O}_{2}$ desaturation decreased slightly, from $97.8 \pm 1.1 \%$ [range 95.5-99.8\%] to $96.1 \pm 1.4 \%$ [range 93.6-98.4\%] after the events $\left(t_{1,26}=17.81, p<0.001\right)$. For apneic events with blood- $\mathrm{O}_{2}$-desaturation, the mean $\mathrm{SpO}_{2}$ fell from $97.8 \pm 0.9 \%$ [range $95.6-100.0 \%$ ] to $91.4 \pm 1.8 \%$ [range $87.8-94.6 \%$ ] $\left(t_{1,25}=20.78, p<0.001\right)$. There was no influence of the sleep state on this drop in $\mathrm{SpO}_{2}$.

When data from all sleep stages were pooled, apneic events with blood- $\mathrm{O}_{2}$-desaturation did not differ from those without

Table 1. Frequency $\left(h^{-1}\right)$, mean and maximum duration $(s)$ of apneic events according to sleep state (active sleep: AS and quiet sleep: $Q S)$ and blood- $\mathrm{O}_{2}$-desaturation state

\begin{tabular}{lcc}
\hline \multicolumn{1}{c}{ Apneic events } & AS & QS \\
\hline Frequency $\left(\mathrm{h}^{-1}\right)$ & & \\
$\quad$ With desaturation & $33.3 \pm 29.2$ & $20.0 \pm 27.3^{*}$ \\
$\quad$ Without desaturation & $28.6 \pm 19.5$ & $24.2 \pm 29.0$ \\
Mean duration (s) & & \\
$\quad$ With desaturation & $6.0 \pm 1.3$ & $7.3 \pm 2.6$ \\
$\quad$ Without desaturation & $4.9 \pm 0.9$ & $5.7 \pm 1.0 \dagger$ \\
Maximum duration (s) & & \\
$\quad$ With desaturation & $8.2 \pm 2.2$ & $8.6 \pm 2.9$ \\
$\quad$ Without desaturation & $7.6 \pm 2.0$ & $7.3 \pm 2.4$ \\
Drop in SpO $(\%)$ & & \\
$\quad$ With desaturation & $6.3 \pm 1.6$ & $6.2 \pm 2.1$ \\
$\quad$ Without desaturation & $1.8 \pm 0.6$ & $1.7 \pm 0.7$ \\
Respiratory rate (breaths $\min ^{-1}$ ) & $56.6 \pm 9.2$ & $53.0 \pm 11.8$ \\
Heart rate (beats min & \\
Hea & $147.3 \pm 7.6$ & $141.2 \pm 10.2 \ddagger$ \\
\hline
\end{tabular}

Values are mean \pm standard deviation, calculated for the infants exhibiting apnea with (or without) desaturation in AS and QS.

$* p<0.05, \dagger p<0.01$, and $\ddagger p<0.001$ between AS and QS. 
desaturation in terms of their frequency $(22.4 \pm 4.1 \mathrm{vs} 25.7 \pm$ $3.3 \mathrm{~h}^{-1}$, respectively, $\mathrm{F}_{1,9}=0.14, \mathrm{NS}$ ) but were longer (mean duration: $6.3 \pm 0.3$ vs $5.2 \pm 1.0 \mathrm{~s}, \mathrm{~F}_{1,9}=7.79$, respectively, $p=0.021$; maximum duration: $8.3 \pm 0.4$ vs $7.5 \pm 0.3 \mathrm{~s}$, respectively, $\mathrm{F}_{1,9}=5.16, p=0.049$ ). The frequency of apneic events with desaturation was higher in AS than in QS $(+13.3$ $\left.\mathrm{h}^{-1}, t_{11}=2.66, p=0.022\right)$. This was not the case for apneic events without blood- $\mathrm{O}_{2}$-desaturation $\left(t_{23}=0.86, \mathrm{NS}\right)$. The mean duration of events without blood- $\mathrm{O}_{2}$-desaturation was longer $\left(t_{23}=3.00, p=0.007\right)$ during QS. This did not reach significance for events with desaturation $\left(t_{11}=1.76, \mathrm{NS}\right)$. The maximum event duration did not differ in AS compared with QS, either for events with desaturation $\left(t_{11}=1.77, \mathrm{NS}\right)$ or events without blood- $\mathrm{O}_{2}$-desaturation $\left(t_{23}=0.60, \mathrm{NS}\right)$.

The respiratory rate did not differ between AS and QS $\left(t_{24}=1.70, \mathrm{NS}\right)$. In contrast, the heart rate was higher in AS than in QS $\left(t_{26}=5.98, p<0.001\right)$.

Regressions were computed for the frequency of apneic events with desaturation, the frequency of apneic events without desaturation and the proportion of apneic events with desaturation, the mean and maximal durations as a function of oxygen consumption and the respiratory parameters measured during the FRC baseline period (Table 2). None of these regressions was significant, except for the overall proportion of apneic events with desaturation $\left(\mathrm{F}_{1,36}=6.24, p=0.017\right.$, $r^{2}=0.148$, Fig. 1) and the frequency of apneic events with desaturation $\left(\mathrm{F}_{1,36}=3.18, p=0.083, r^{2}=0.081\right)$ relative to minute ventilation: the lower the $\dot{V}_{E}$ value, the higher the proportion and frequency of apneic events with desaturation.

Although the regression calculated between the proportion of apneic events with desaturation and the baseline level of $\mathrm{SpO}_{2}$ failed to reach significance (Table 2), unpaired comparisons between neonates with low blood $\mathrm{O}_{2}$ saturation values during the baseline phase and neonates with high values (where the median value of $97.8 \%$ was the cut-off) indicated that the proportion (but not frequency) of apneic events with desaturation tended to be higher in the first group (proportion: $0.50 \pm 0.28$ vs $0.34 \pm 0.20$, respectively, $t_{37}=1.98, p=$ 0.05; frequency: $26.67 \pm 28.97$ vs $15.86 \pm 20.11 \mathrm{~h}^{-1}, t_{37}=$ 1.24 , NS).

$\boldsymbol{F R C}$. The intrasubject coefficient of variation for FRC was $13.7 \pm 11.1 \%$. The overall mean value of the FRC was $31.2 \pm$ $9.6 \mathrm{~mL} \mathrm{~kg}^{-1}$. The difference between the sleep states did not reach significance (AS: $30.7 \pm 8.3 v s$ QS: $31.8 \pm 11.0 \mathrm{~mL}$ $\left.\mathrm{kg}^{-1}, t_{23}=1.06, \mathrm{NS}\right)$.
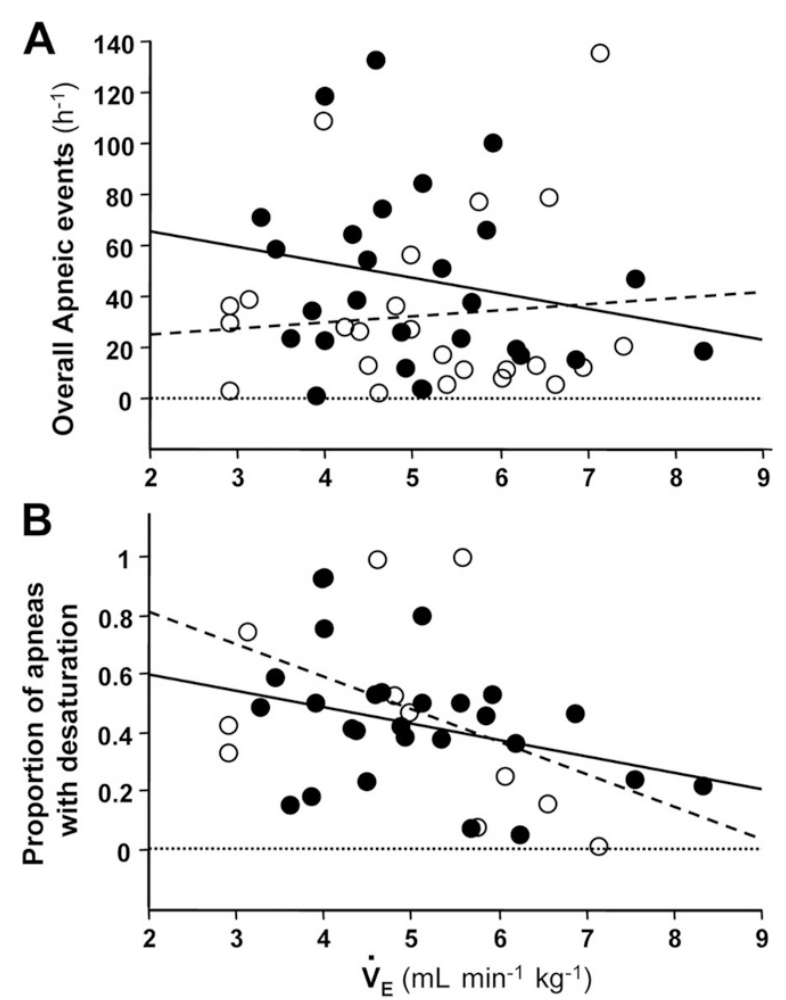

Figure 1. Relationships between the frequency of apneic events $\left(\mathrm{h}^{-1}\right.$, panel A) and the proportion of apneic events with blood- $\mathrm{O}_{2}$-desaturation (panel B) as a function of minute ventilation $\left(\dot{V}_{E}, \mathrm{~mL} \min ^{-1} \mathrm{~kg}^{-1}\right)$, for each sleep state. The correlation between the overall frequency of apneic events and $\dot{V}_{E}$ failed to reach significance (all sleep states pooled: $F_{1,36}=3.18$, NS; active sleep, AS, $\bullet$, solid line: $\mathrm{F}_{1,25}=1,21$, NS; quiet sleep, QS, $\circ$, dotted line: $\left.\mathrm{F}_{1,9}=0.20, \mathrm{NS}\right)$. In contrast, the overall proportion of apneic events with blood- $\mathrm{O}_{2}$-desaturation (panel B) was correlated with $\dot{V}_{E}\left(\mathrm{~F}_{1,36}=6.24, p=\right.$ $\left.0.017, r^{2}=0.148\right)$. When considering the sleep state, the correlation showed a trend to significance for $\mathrm{AS}\left(\mathrm{F}_{1,25}=3.03, p=0.09, r^{2}=\right.$ $0.112)$ but not for QS $\left(F_{1,9}=2.502, \mathrm{NS}\right)$.

There was a negative relationship between the frequency of apneic events and FRC (frequency $=76.87-1.143 \times$ FRC; $\left.\mathrm{F}_{1,48}=4.34, p=0.043, r^{2}=0.083\right)$, i.e., the lower the FRC value, the higher the frequency of apneic episodes (Fig. 2). This relationship was borderline significant during $\operatorname{AS}\left(\mathrm{F}_{1,26}=\right.$ 4.17, $p=0.051, r^{2}=0.138$ ) but not significant during QS $\left(\mathrm{F}_{1,20}=1.11, \mathrm{NS}\right)$. FRC value was positively related to $\mathrm{SpO}_{2}$ value during the baseline period $\left(\mathrm{F}_{1,50}=4.38, p=0.042\right)$.

When analyzed according to the type of apnea, no correlation was found between FRC on one hand and apneic events

Table 2. Regression between the respiratory parameters and the proportion of apneic events with blood $\mathrm{O}_{2}$-desaturation

\begin{tabular}{lcc}
\hline & Overall mean values $\pm \mathrm{SD}$ & Regression with the proportion of apneic events with blood $\mathrm{O}_{2}$-desaturation \\
\hline$\dot{V}_{E}\left(\mathrm{~mL} \mathrm{~min} \mathrm{~min}^{-1} \mathrm{~kg}^{-1}\right)$ & $5.1 \pm 1.3$ & $\mathrm{~F}_{1,36}=6.24, p=0.017, r^{2}=0.148($ Kendall: $\mathrm{Z}=2.32, p=0.021)$ \\
$V_{\mathrm{t}}(\mathrm{mL})$ & $5.2 \pm 1.2$ & $\mathrm{NS}$ \\
$\mathrm{BR}\left(\mathrm{breaths} \mathrm{min}^{-1}\right)$ & $54.3 \pm 9.6$ & $\mathrm{NS}$ \\
$T_{\mathrm{i}}(\mathrm{s})$ & $0.48 \pm 0.08$ & $\mathrm{NS}$ \\
$T_{\mathrm{e}}(\mathrm{s})$ & $0.66 \pm 0.16^{*}$ & $\mathrm{NS}$ \\
$\mathrm{Spo}$ & $97.7 \pm 1.5$ & $\mathrm{~F}_{1,36}=2.90, p=0.098, r^{2}=0.076($ Kendall: $\mathrm{Z}=1.80, p=0.072)$ \\
$\dot{V}_{O_{2}}\left(\mathrm{~mL} \min ^{-1} \mathrm{~kg}^{-1}\right)$ & $7.1 \pm 1.8^{*}$ & $\mathrm{NS}$ \\
\hline
\end{tabular}

$\dot{V}_{E}=$ minute ventilation $\left(\mathrm{mL} \min ^{-1} \mathrm{~kg}^{-1}\right) ; V_{\mathrm{t}}=$ tidal volume $(\mathrm{mL}) ; \mathrm{BR}=$ breath rate (breaths $\left.\mathrm{min}^{-1}\right), T_{\mathrm{i}}$ and $T_{\mathrm{e}}=$ inspiratory and expiratory time $(\mathrm{s})$, $\mathrm{SpO}_{2}=$ blood $\mathrm{O}_{2}$ saturation $(\%) ; \dot{V}_{O_{2}}=$ oxygen consumption $\left(\mathrm{mL} \min ^{-1} \mathrm{~kg}^{-1}\right)$.

$* p<0.001$ between AS and QS. 

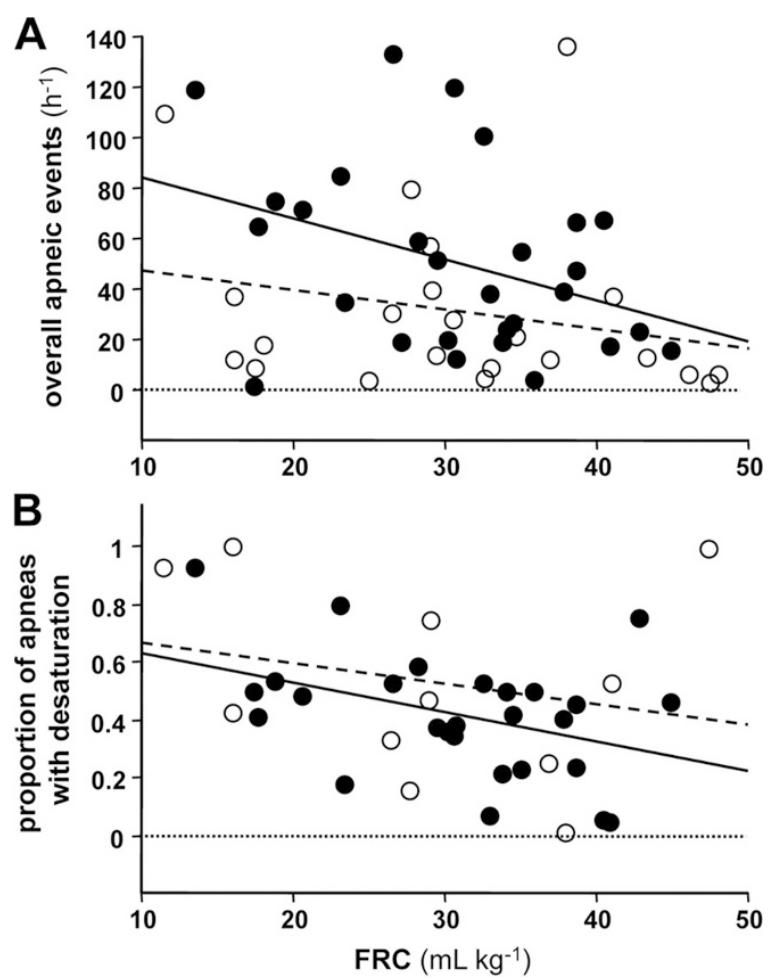

Figure 2. Relationship between the frequency of apneic events $\left(\mathrm{h}^{-1}\right.$, panel A) and the proportion of apneic events with blood- $\mathrm{O}_{2}$-desaturation (panel B) as a function of functional residual capacity (FRC, $\mathrm{mL} \mathrm{kg}^{-1}$ ), for each sleep state. The correlation between the overall frequency of apneic events and FRC was significant $\left(\mathrm{F}_{1,48}=4.34, p=0.043, r^{2}=0.083\right)$. When considering the sleep state, this correlation was significant in active sleep (AS, $\bullet$, solid line; $\left.\mathrm{F}_{1,26}=4.17, p=0.051, r^{2}=0.138\right)$ but not in quiet sleep $(\mathrm{QS}, \circ$, dotted line; $\mathrm{F}_{1,20}=1.11$, NS). The proportion of apneic events with blood- $\mathrm{O}_{2^{-}}$ desaturation was correlated with FRC $\left(\mathrm{F}_{1,36}=4.52, p=0.041, r^{2}=0.111\right)$. When considering the sleep state, this correlation was significant in AS $\left(\mathrm{F}_{1,25}=4.78, p=0.038, r^{2}=0.160\right)$ but not in QS $\left(\mathrm{F}_{1,9}=0.519\right.$, NS $)$.

without blood- $\mathrm{O}_{2}$-desaturation on the other $\left(\mathrm{F}_{1,46}=0.10, \mathrm{NS}\right.$, Kendall's correlation coefficient: $\mathrm{Z}=0.36$, NS). In contrast, there was a significant correlation between FRC and the frequency of apneic events with desaturation $\left(\mathrm{F}_{1,36}=15.05\right.$, $p<0.001, r^{2}=0.295$; Kendall: $\left.\mathrm{Z}=2.83, p=0.005\right)$ : the lower the FRC, the higher the frequency of apneic events with desaturation. The regression lines were similar in both AS and QS, although the probability did not reach significance for the latter (AS: $\mathrm{F}_{1,25}=10.07, p=0.004, r^{2}=0.287$; QS: $\mathrm{F}_{1,9}=$ $\left.4.70, p=0.058, r^{2}=0.343\right)$. Similarly, the proportion of apneic events with desaturation was correlated with FRC values (Fig. 2, $\left.\mathrm{F}_{1,36}=4.52, p=0.041, r^{2}=0.111\right)$. In contrast to AS, this result was not significant in QS (AS: $\mathrm{F}_{1,25}=4.78, p=0.038, r^{2}=$ $0.160 ;$ QS: $\mathrm{F}_{1,9}=0.52$, NS).

No relationship was found between FRC and the mean duration of apneic events (with or without blood- $\mathrm{O}_{2}$ desaturation: $\mathrm{F}_{1,36}=0.10$ and $\mathrm{F}_{1,46}=0.19$, NS) or the maximum episode duration $\left(\mathrm{F}_{1,36}=1.73\right.$ and $\mathrm{F}_{1,46}=0.53$, NS).

\section{DISCUSSION}

The present study shows that lower baseline $\dot{V}_{E}$ and $\mathrm{SpO}_{2}$ values are positively related to the proportion of apneic events with desaturation (pooled but not when sleep states are considered alone), explaining $\sim 15 \%$ (significant) and $\sim 8 \%$ (indicative) of the variability. This was not observed for apneic events without desaturation. Baseline oxygen consumption and heart rate were increased in AS but without any relationship with the proportion of apneic events with desaturation. Overall and in AS but not QS, lower FRC values are related to the frequency, but not to the duration, of all apneic events, explaining $\sim 14 \%$ of the variability. The proportion of brief apneic events with desaturation is correlated with FRC in AS, explaining $\sim 16 \%$ of the variability. During QS, in comparison with AS, the duration of brief apneic events without desaturation is increased.

The frequency of apneic events is similar to previous data reported for the same population and methods (22). Apneic events without blood- $\mathrm{O}_{2}$-desaturation were more frequent in AS than in QS. Apneic events with desaturation were not more frequent but had greater mean and maximum durations than those without blood- $\mathrm{O}_{2}$-desaturation. Unfortunately, the low number of long-lasting apneic episodes in our population prevented us from analyzing our data according to the duration of the events and extrapolating our results to pathologic apnea.

FRC measurements. FRC was assessed using the oxygen wash-in method (19), which is well correlated with the $\mathrm{He}$ dilution technique (20). The $\mathrm{O}_{2}$-technique can overestimate FRC by about $10 \%$ when the tidal volume is low but is highly reproducible (19). Hence, bearing in mind this systematic overestimation, our overall mean FRC value of $31.2 \pm 9.6 \mathrm{~mL}$ $\mathrm{kg}^{-1}$ is within the wide range reported in previous studies (using a variety of other methods) of infants of similar ages and body masses $(7,23)$. Part of the difference with previous studies may result from different techniques and populations. Although we took care to limit variability, the intrasubject coefficient of variation for FRC was $13.7 \pm 11.1 \%$, which corresponds to somewhat lower reproducibility than previously reported with the He technique (8\%) (4). Greenough et al. (4) also obtained a wide range of FRC values (healthy neonates: $20.7-35.2 \mathrm{~mL} \mathrm{~kg}^{-1}$; neonates with bronchopulmonary dysplasia: $13.2-29.5 \mathrm{~mL} \mathrm{~kg}^{-1}$ ).

We failed to observe a sleep state effect on FRC values as previously reported $(7,11,23,24)$, probably because we only measured FRC during regular breathing pattern. In contrast, less recent studies found that the FRC was lower in AS (determined by the behavior and/or from electro-oculograms but without EEG data) $(2,10)$. Moriette et al. (7) suggested that FRC was lower during irregular breathing than during regular breathing. Poets et al. (3) confirmed a lower FRC in irregular breathing. Hence, differences in breathing pattern regularity may better account for the above-reported FRC differences (and the higher frequency and proportion of apneic episodes with desaturation during AS) than a specific sleep state effect per se.

We observed that hypoventilation (i.e., $\dot{V}_{E}$ ) and low levels of baseline blood $\mathrm{O}_{2}$ saturation are related to apneic events with desaturation. The frequency and the proportion of apneic events with desaturation increase with hypoventilation (Fig. $1)$. The proportion of apneic events with blood- $\mathrm{O}_{2}-$ 
desaturation increases with lower baseline blood $\mathrm{O}_{2}$-saturation measured just before the apneic event. This confirms previous data (25). As a result, hypoventilation and low basal blood $\mathrm{O}_{2}$-saturation values are associated with low $\mathrm{O}_{2}$ reserves and may increase the likelihood of blood $\mathrm{O}_{2}$-desaturation-even during short apneic events. There was no difference between the two types of event in terms of inspiratory and expiratory times, tidal volume, and breathing rate (i.e., all parameters describing changes in pulmonary resistance and compliance).

FRC and overall apneic events. We found that FRC was negatively correlated with the frequency of overall apneic events during AS but not during QS. Although a decrease in lung volume following frequent apneic episodes or irregular breathing periods has been observed $(3,23,24)$; this is the first time (to the best of our knowledge) that a sleep-state dependent relationship has been established between FRC and frequent, short apneic events.

Poets et al. (3) have reported that apneic events decrease the FRC value measured during the following 2 min whereas a sigh redistributes pulmonary volume and reverses the apneainduced decrease in FRC. Our results, pointing out that the FRC value is related to the overall apneic event frequency (i.e., measured over the entire 3 -h experiment), confirm that the apnea-induced FRC decrease lasts several minutes. The absence of a significant relationship with the mean duration of apneic events has been described previously (3).

Low FRC may be related to low arterial $\mathrm{Po}_{2}$ values in neonates. Although we measured $\mathrm{SpO}_{2}$ only, the latter parameter is thought to accurately reflect the simultaneous $\mathrm{PaO}_{2}$ (26); in our work, $\mathrm{SpO}_{2}$ was negatively correlated with FRC. Recent studies $(27,28)$ have reported that a low $\mathrm{PaO}_{2}$ magnifies the peripheral chemoreceptors' contribution to breathing and induces major ventilatory changes. As a result, respiratory instability and increased apnea frequency may be observed. These findings are consistent with the "overshoot/undershoot" concept (29) explaining periodic breathing and according to which increased carotid body activity can promote respiratory instability and apnea when the $\mathrm{PCO}_{2}$ falls below the apneic threshold (27). The greater tonic activity of the peripheral chemoreceptors in AS than in QS (30) may exaggerate this phenomenon during the former sleep state.

There is a significant relationship between FRC and the frequency of the apneic events during AS but not during QS. This sleep state difference is difficult to explain and would require further investigation of respiratory mechanics, activity of the respiratory muscles and/or the presence of alveolar shunts after end-expiratory apnea.

FRC and apneic events with desaturation. Our results on the proportion of apneic events with desaturation indicate that lower FRC is associated with an increased likelihood of desaturation during an apneic event. Only Poets et al. (3) have clearly demonstrated that FRC was negatively related to the speed of blood- $\mathrm{O}_{2}$-desaturation within $2 \mathrm{~min}$ of the FRC measurement; however, they did not consider both apneic events and desaturation.

In the case of a low FRC, the lung's end expiratory volume is close to the closing volume. During apnea, the closure of a few regions of the lung could occur and induce intrapulmo- nary shunting. A proportion of the desaturated blood circulating through the shunting area would not be oxygenated and would return to the peripheral circulation, inducing the rapid transcutaneous desaturation that can be observed during the apnea (6). Such a mechanism would be consistent with our results, because the lower FRC was observed with more frequent, short apneic events associated with blood- $\mathrm{O}_{2}-$ desaturation.

Sleep state and respiratory processes. The sleep state difference in the relationship between apneic events with desaturation on one hand and the FRC value on the other is difficult to explain, given the absence of a significantly lower FRC value during AS. However, the sleep state effect seems to be inconsistent; there were similar results in AS and QS for the frequency of apneic events with desaturation, whereas the relationship between the proportion of these events and the FRC value is not significant during QS. We could speculate that the influence of FRC is overridden by specific, AS-related mechanisms.

Our results suggest that mechanisms other than FRC may be involved in the occurrence of desaturation during apnea. Indeed, for equivalent FRC values, the frequency and proportion of apneic events with desaturation vary from one infant to another: the relationship between the proportion of apneic events with desaturation and FRC only accounts for $16.0 \%$ of the observed variance during AS. This event does not seem to be linked to pulmonary mechanics (as observed above).

The higher frequency of apneic events in general (and those with desaturation in particular) observed in AS cannot solely be explained by a reduction in FRC during this sleep state. The fact that our protocol excluded irregular breathing could explain this finding but there is also evidence to suggest that arterial blood oxygenation is lower and more variable during this sleep state (31). Likewise, the spontaneous hypoxemia threshold is lower and the $\mathrm{PaCO}_{2}$ threshold for apnea can cause breathing instability. The genesis of apneic events and desaturation during those events is multifactorial; AS versus QS differences in peripheral chemoreceptor activity and/or impairment of pulmonary diffusion (e.g., decreases in the alveolar partial pressure of $\mathrm{O}_{2}$ ) may contribute to the pathogenesis of such events without any relationship with lung volume changes (30). Interestingly, the duration of short apneic events appears not to be subject to the same mechanisms, although this needs to be confirmed with longer, pathologic apneic episodes.

In conclusion, in late preterm infants the frequency (but not the duration or proportion) of short apneic events with desaturation are AS-related with minor relationships with ventilation/oxygenation/FRC suggesting that desaturation is more likely to occur with apneic events when these parameters have lower baseline values in AS, a time when heart rate and oxygen consumption are increased. However, brief apneic events without desaturation are not related to these parameters in AS or QS, despite a longer duration of brief central apnea without desaturation in QS. Thus, it is hard to speculate that the AS-linked relationships reported above are signaled by FRC-mediated chemical changes and related to cycles of hypo- and hyperventilation unless it can be demonstrated that 
such changes are only triggered/only occur in AS. It appears that, at least at baseline, respiratory parameter differences cannot explain the AS influence, nor can lower baseline values of FRC.

Acknowledgments. The authors thank Stéphane Delanaud, David Fraser, the staff of the NICU and the neonates and parents who participated in the study.

\section{REFERENCES}

1. Newth CJ, Enright P, Johnson RL 1997 Multiple-breath nitrogen washout techniques: including measurements with patients on ventilators. Eur Respir J 10:21742185

2. Lopes J, Muller NL, Bryan MH, Bryan AC 1981 Importance of inspiratory muscle tone in maintenance of FRC in the newborn. J Appl Physiol 51:830-834

3. Poets CF, Rau GA, Neuber K, Gappa M, Seidenberg J 1997 Determinants of lung volume in spontaneously breathing preterm infants. Am J Respir Crit Care Med 155:649-653

4. Greenough A, Dimitriou G, Bhat RY, Broughton S, Hannam S, Rafferty GF, Leipala JA 2005 Lung volumes in infants who had mild to moderate bronchopulmonary dysplasia. Eur J Pediatr 164:583-586

5. Thibeault DW, Wong MM, Auld PA 1967 Thoracic gas volume changes in premature infants. Pediatrics 40:403-411

6. Poets CF, Samuels MP, Southall DP 1992 Potential role of intrapulmonary shunting in the genesis of hypoxemic episodes in infants and young children. Pediatrics 90:385-391

7. Moriette G, Chaussain M, Radvanyi-Bouvet MF, Walti H, Pajot N, Relier JP 1983 Functional residual capacity and sleep states in the premature newborn. Biol Neonate 43:125-133

8. Martin RJ, Abu-Shaweesh JM 2005 Control of breathing and neonatal apnea. Biol Neonate 87:288-295

9. Marshall TA, Kattwinkel J 1981 Functional residual capacity and oxygen tension in apnea of prematurity. J Pediatr 98:479-482

10. Henderson-Smart DJ, Read DJ 1979 Reduced lung volume during behavioral active sleep in the newborn. J Appl Physiol 46:1081-1085

11. Beardsmore CS, MacFadyen UM, Moosavi SS, Wimpress SP, Thompson J, Simpson H 1989 Measurement of lung volumes during active and quiet sleep in infants. Pediatr Pulmonol 7:71-77

12. Darnall RA, Ariagno RL, Kinney HC 2006 The late preterm infant and the control of breathing, sleep, and brainstem development: a review. Clin Perinatol 33:883-914

13. Bach V, Telliez F, Libert JP 2002 The interaction between sleep and thermoregulation in adults and neonates. Sleep Med Rev 6:481-492
14. Telliez F, Bach V, Delanaud S, Bouferrache B, Krim G, Libert JP 1997 Skin derivative control of thermal environment in a closed incubator. Med Biol Eng Comput 35:521-527

15. Curzi-Dascalova L, Mirmiran M, eds 1996 Manual of Methods for Recording and Analyzing Sleep-Wakefulness States in Preterm and Full-Term Infant. Paris: Les Editions INSERM, pp 1-162

16. Berterottiere D, D'Allest AM, Dehan M, Gaultier C 1990 Effects of increase in body temperature on the breathing pattern in premature infants. J Dev Physiol 13:303-308

17. Franco P, Szliwowski H, Dramaix M, Kahn A 2000 Influence of ambient temperature on sleep characteristics and autonomic nervous control in healthy infants. Sleep 23:401-407

18. 1996 Standards and indications for cardiopulmonary sleep studies in children American Thoracic Study. Am J Respir Crit Care Med 153:866-878

19. Weismann D, Reissmann H, Maisch S, Fullekrug B, Schulte J 2006 Monitoring of functional residual capacity by an oxygen washin/washout; technical description and evaluation. J Clin Monit Comput 20:251-260

20. Mitchell RR, Wilson RM, Holzapfel L, Benis AM, Sierra D, Osborn JJ 1982 Oxygen wash-in method for monitoring functional residual capacity. Crit Care Med 10:529533

21. Bouferrache B, Filtchev S, Léké A, Marbaix-Li Q, Fréville M, Gaultier C 2000 The hyperoxic test in infants reinvestigated. Am J Respir Crit Care Med 161:160-165

22. Curzi-Dascalova L, Christova-Gueorguieva E 1983 Respiratory pauses in normal prematurely born infants. A comparison with full-term newborns. Biol Neonate 44:325-332

23. Walti H, Moriette G, Radvanyi-Bouvet MF, Chaussain M, Morel-Kahn F, Pajot N, Relier JP 1986 Influence of breathing pattern on functional residual capacity in sleeping newborn infants. J Dev Physiol 8:167-172

24. Stokes GM, Milner AD, Newball EA, Smith NJ, Dunn C, Wilson AJ 1989 Do lung volumes change with sleep state in the neonate? Eur J Pediatr 148:360-364

25. Adams JA, Zabaleta IA, Sackner MA 1997 Hypoxemic events in spontaneously breathing premature infants: etiologic basis. Pediatr Res 42:463-471

26. Hay WW Jr, Brockway JM, Eyzaguirre M 1989 Neonatal pulse oximetry: accuracy and reliability. Pediatrics 83:717-722

27. Cardot V, Chardon K, Tourneux P, Micallef S, Stephan E, Leke A, Bach V, Libert JP, Telliez F 2007 Ventilatory response to a hyperoxic test is related to the frequency of short apneic episodes in late preterm neonates. Pediatr Res 62:591-596

28. Al-Matary A, Kutbi I, Qurashi M, Khalil M, Alvaro R, Kwiatkowski K, Cates D, Rigatto H 2004 Increased peripheral chemoreceptor activity may be critical in destabilizing breathing in neonates. Semin Perinatol 28:264-272

29. Cherniack NS, Longobardo GS, Levine OR, Mellins R, Fishman AP 1966 Periodic breathing in dogs. J Appl Physiol 21:1847-1854

30. Chardon K, Bach V, Telliez F, Tourneux P, Elabbassi EB, Cardot V, Gaultier C, Libert JP 2003 Peripheral chemoreceptor activity in sleeping neonates exposed to warm environments. Neurophysiol Clin 33:196-202

31. Martin RJ, Okken A, Rubin D 1979 Arterial oxygen tension during active and quie sleep in the normal neonate. J Pediatr 94:271-274 